\title{
Bioakkumulation von Metallen und Stickstoff zwischen 1990 und 2005 in Niedersachsen
}

\author{
Karsten Mohr $\cdot$ Marcel Holy $\cdot$ Roland Pesch $\cdot$ Winfried Schröder
}

Eingegangen: 16. Juni 2009/Akzeptiert: 24. August 2009/Online veröffentlicht: 22. September 2009

(C) Springer-Verlag 2009

Zusammenfassung Hintergrund, Ziel und Bereich Das Moosmonitoring liefert seit 1990 alle fünf Jahre flächendeckende Inventuren der Schwermetallbioakkumulation in Europa. Seit 2005 wird auch die immissionsbedingte Stickstoffanreicherung erfasst. In Deutschland wird das Monitoring von Bund und Ländern gemeinsam durchgeführt. Folglich ist neben der bundesweiten auch eine länderspezifische Auswertung von Interesse. Dieser Artikel behandelt die Kartierung der zeitlichen Entwicklung der Metallanreicherung in Niedersachsen seit 1990, die Stickstoffanreicherung 2005 sowie die räumliche Varianz der Metallbioakkumulation in Abhängigkeit von Eigenschaften der Probeentnahmestellen und ihrer Umgebung.

Material und Methoden Die Anreicherung von bis zu 40 Spurenelementen und Stickstoff in Moosen wurde nach europaweit harmonisierter Vorgehensweise ermittelt. Die hierbei berücksichtigte Richtlinie regelt die Auswahl der Moosprobensammelorte und der Moosarten, ihre chemische Analyse einschließlich der Qualitätskontrolle sowie die Klassierung der Messdaten für ihre Kartierung. Für Niedersachsen wurden die bis zu 130 beprobten Standorte hinsichtlich topografischer und ökologischer Merkmale so-

\section{K. Mohr}

Landwirtschaftskammer Niedersachsen, FB 3.1.11,

Mars-la-Tour-Straße 1-13, 26121 Oldenburg, Deutschland

E-Mail: karsten.mohr@1wk-niedersachsen.de

M. Holy $\cdot$ R. Pesch $\cdot$ W. Schröder $(\bowtie)$

Lehrstuhl für Landschaftsökologie, Hochschule Vechta,

PF 1553, 46364 Vechta, Deutschland

E-Mail: wschroeder@iuw.uni-vechta.de

M. Holy

E-Mail: mholy@iuw.uni-vechta.de

R. Pesch

E-Mail: rpesch@iuw.uni-vechta.de wie Informationen über die Landnutzung in der Umgebung der Moosprobenentnahmeorte mit den Messdaten in dem WebGIS MossMet zusammengeführt und ausgewertet: Die räumliche Struktur der Metall- und Stickstoffanreicherung wurde per Variogrammanalyse untersucht und modelliert sowie mit Kriging-Interpolation flächenhaft dargestellt. Aus den standort- und metallspezifischen Messdaten sowie den daraus geostatistisch berechneten Flächendaten über die Metallakkumulation wurde ein zusammenfassender Multimetallindex jahresübergreifend $\left(\mathrm{MMI}_{1990-2005}\right)$ für $\mathrm{As}, \mathrm{Cd}$, $\mathrm{Cr}, \mathrm{Cu}, \mathrm{Fe}, \mathrm{Ni}, \mathrm{Pb}, \mathrm{Ti}, \mathrm{V}$ und $\mathrm{Zn}$ berechnet und kartiert. Die Zusammenhänge zwischen den Schwermetall- und N-Akkumulationen, Standortcharakteristika sowie Landnutzung und Emissionen wurden mit Rangkorrelationskoeffizienten und Kontingenztafeln sowie am Beispiel von Sb multivariatstatistisch mit Classification and Regression Trees (CART) quantifiziert.

Ergebnisse Die Ergebnisse der qualitätskontrollierten chemischen Analysen zeigen von 1990 bis 2000 einen statistisch signifikanten Rückgang der Bioakkumulation für die meisten Metalle. Von 2000 bis 2005 ist z. T. ein Anstieg der Metallbioakkumulation zu beobachten. Er fällt am höchsten bei $\mathrm{Cr}$ aus und ist auch statistisch signifikant, am niedrigsten bei $\mathrm{Fe}$ und betrifft ansonsten $\mathrm{Cu}, \mathrm{Sb}$ und $\mathrm{Zn}$ (alle nicht signifikant). Die Anreicherung von Cd nahm von 2000 bis 2005 signifikant ab, der Rückgang von As ist hingegen nicht signifikant. Eine elementübergreifende Darstellung liefert der MMI: Nachdem dieser von 1990 bis 2000 signifikant zurückging, stieg er von 2000 bis 2005 signifikant an. Die in den niedersächsischen Moosen gemessenen Stickstoffwerte rangieren zwischen 1,1 und 1,9\% in der Trockenmasse. Hohe N-Konzentrationen wurden in agrarisch intensiv genutzten Gebieten festgestellt. Hoch signifikante bivariate Rangkorrelationen zwischen den Metallgehalten und der Landnutzung im Umkreis der Moossammelorte wurden mit 
Werten zwischen 0,3 und 0,5 nachgewiesen. Von den Standortmerkmalen weisen vor allem die Variablen Moosart, orografische Höhe, Meeresdistanz und Entfernung der Moosentnahmestelle von Baumkronen entsprechend deutliche Korrelationen zur Metallakkumulation auf. Stickstoff lässt in der bivariaten Korrelationsstatistik nur negative Zusammenhänge mit dem urbanen Flächenanteil in der Umgebung der Beprobungsorte sowie mit der Entfernung zu Baumkronen erkennen. Die am Beispiel von den Sb-Konzentrationen 2005 untersuchten multivariat-statistischen Korrelationen weisen auf Interaktionen von Moosart sowie den Flächenanteilen agrarischer, forstlicher und urbaner Nutzung hin.

Diskussion Der in den vergangenen 15 Jahren festgestellte kontinuierliche Rückgang der Schwermetallgehalte in den Moosen spiegelt die allgemein verbesserte Immissionssituation in Niedersachsen wider. Von diesem Trend ausgenommen sind nur wenige Stoffe wie z.B. Cr. Dieser Befund steht im Gegensatz zu anderen Monitoringdaten. Insbesondere der Anstieg der Cr-Bioakkumulation vom Jahr 2000 zum Jahr 2005 wurde auch in anderen Teilnehmerstaaten des europaweiten Moosmonitorings wie z. B. in der Schweiz bestätigt. Es bedarf weiterer Untersuchungen, $o b$ es sich hierbei um einen emissionsbedingten oder einen biogenen Effekt (z.B. infolge gleichzeitig angestiegener Stickstoffbelastung) handelt. Im Vergleich zu zeitlich höher aufgelösten Depositionsmessungen erfasst das Moosmonitoring großräumig ein breiteres Stoffspektrum, das auch in anderen Messnetzen selten gemessene Stoffe mit humantoxikologischer Bedeutung (z. B. As, Al, Hg, Sb, V) umfasst. Mit dem 2005 einbezogenen Stickstoff wird erstmalig eine ökotoxikologisch wichtige Stoffgruppe berücksichtigt, für die - im Unterschied zu den Schwermetallen - in Niedersachsen regional hohe Immissionsbelastungen festzustellen sind.

Das standardisierte Biomonitoring von Luftverunreinigungen mit Bodenmoosen bildet ein wichtiges Bindeglied zwischen der technischen Erfassung von Stoffeinträgen und der Anreicherung dieser Stoffe in biologischem Material. Die häufig zum Qualitätsnachweis für das Moosmonitoring erhobene Forderung, die Stoffkonzentrationen im biologischen Material müsse mit der gemessenen oder modellierten Deposition hoch korrelieren, ist nicht sachgemäß, da es sich um Verfahren handelt, die unterschiedliche Rezeptoren berücksichtigen. Die Akkumulation der untersuchten Schadstoffe in den Bodenmoosen reflektiert die im System „Pflanze - Boden“ den durch Depositionen hervorgerufenen, verbleibenden und wirksamen Teil der Schadstoffbelastungen. Der Betrag ihrer statistischen Korrelation hängt ab von der Ausprägung der Randbedingungen der physikalischen Prozesse, so u. a. von den regional und standörtlich im Anreicherungszeitraum vorherrschenden meteorologischen Verhältnissen, der horizontalen und vertikalen Vegetationsstruktur und der Landnutzung.
Schlussfolgerungen Das Moosmonitoring liefert wesentliche Beiträge zum Schwermetall- und zum Multikomponentenprotokoll der CLRTAP. Es belegt flächendeckend, wie sich Luftreinhaltepolitik auf die Anreicherung von atmosphärischen Stoffeinträgen in Schutzgütern wie der Vegetation (einschließlich Nahrungspflanzen) auswirkt. Von besonderer umweltpolitischer Bedeutung ist, dass in keinem anderen Messprogramm räumlich so verdichtet Daten über ein breites, ökotoxikologisch und humanmedizinisch bedeutsames Stoffspektrum erhoben werden. Die räumliche Auflösung von Umweltinformationen ist ein wesentliches Kriterium für ihre Nutzbarkeit im Vollzug umweltpolitischer Maßnahmen auf Bundes- und Länderebene.

Empfehlungen und Perspektiven Das Moosmonitoring ist ein stringent organisiertes Programm der Umweltbeobachtung, das über drei räumliche und administrative Ebenen - Region (z.B. Bundesland oder Naturraum), Staat (z.B. Deutschland) und Kontinent (Europa) methodisch abgestimmte, qualitätskontrollierte Daten über die Stoffanreicherung in der Umwelt liefert und über ein internetfähiges WebGIS-Portal bereitstellt, das die Anforderungen von PortalU und INSPIRE erfüllt.

Schlüsselwörter Bioakkumulation · CART (Classification and Regression Trees) · CHAID (Chisquare Automatic Interaction Detection) ' Geostatistik · Luftschadstoffe · Moosmonitoring $\cdot$ Schwermetalle $\cdot$ Stickstoff $\cdot$ Umweltkonzentration

\section{Trends of metal bioaccumulation from 1990 to 2005 in Germany}

Abstract Background, aim, and scope Since 1990 the UN ECE Heavy Metals in Mosses Surveys provide data inventories of the atmospheric heavy metal bioaccumulation across Europe. In the survey 2005 the nitrogen accumulation was measured for the first time in most of the participating countries. In Germany, the surveys were conducted in close cooperation of the relevant authorities of both the Federal Republic and the sixteen states. Therefore, statistical evaluations of the moss survey data with regard to the whole German territory and single federal states are of interest. This article concentrates on Lower Saxony, dealing with the mapping of the spatiotemporal trends of metal accumulation from 1990 to 2005, the spatial patterns of nitrogen accumulation in 2005 , and the spatial variability of bioaccumulation due to characteristics of the sampling sites and their surroundings.

Materials and methods The bioaccumulation of up to 40 trace elements and nitrogen in mosses was determined according to a Europe-wide harmonised methodology. The according experimental protocol regulates the selection 
of sampling sites and moss species, the chemical analysis and quality control and the classification of the measured values for mapping spatial patterns. In Lower Saxony all sampling sites were described with regard to topographical and ecological characteristics and several criteria to be fulfilled according to the guideline. Together with the measurements this metadata was combined with other information regarding land use in the surroundings of the sampling sites in the WebGIS MossMet. The spatial structure of the metal bioaccumulation was analysed and modelled by variogram analyses and then mapped by applying different Kriging techniques. Furthermore, multi metal indices (MMI) were derived for both the sampling sites and raster maps with help of percentile statistics: The $\mathrm{MMI}_{1990-2005}$ was calculated for $\mathrm{As}, \mathrm{Cd}, \mathrm{Cr}, \mathrm{Cu}, \mathrm{Fe}, \mathrm{Ni}, \mathrm{Pb}, \mathrm{Ti}, \mathrm{V}$ and $\mathrm{Zn}$. The statistical association of the metal and nitrogen bioaccumulation, site specific characteristics as well as information on land use and emissions was analysed by bivariate nonparametric correlation analysis, contingency tables and Classification and Regression Trees (CART).

Results The results of the quality controlled chemical analyses shows a significant decrease of the metal bioaccumulation in Germany from 1990 to 2000 for all elements but Zn. From 2000 to 2005 a further significant decrease can be stated for $\mathrm{Cd}, \mathrm{Hg}$ and $\mathrm{Pb}$, to most parts non significant increases can be observed for $\mathrm{Cr}, \mathrm{Cu}, \mathrm{Fe}, \mathrm{Sb}$ and $\mathrm{Zn}$. $\mathrm{Cr}$ thereby exhibits the highest accumulation in 2005 when compared to the results of 1990, 1995 and 2000. The MMI illustrates the temporal trend of the metal bioaccumulation as a whole: From 1990 to 2000 a continuous significant decrease can be observed. From 2000 to 2005 the median of the MMI increases, again significantly, from 3.4 to 4 . The N concentration in mosses in Lower Saxony reaches from 1.1 to $1.9 \%$ in dry mass. High $\mathrm{N}$ concentrations were detected in agriculturally intensively used areas. Highly significant bivariate correlations between the metal bioaccumulation and land use in the surroundings of the sampling sites were found reaching from 0.3 to 0.5 . Other location criteria with similar correlation coefficients/Cramér's V are moss species, altitude, distance to the North and Baltic Sea and the distance of the sampling site to the nearest tree crown. $\mathrm{N}$ only shows negative correlations to urban land use and the distance to the nearest tree. Exemplified for Sb multivariate correlations were furthermore detected by CART. It could be shown that the $\mathrm{Sb}$ bioaccumulation interacts with the moss species and the ratio of agriculture, forests and urban areas around the sampling site.

Discussion The decrease of the continuously decrease of heavy metals reflects the improving air quality in the past 15 years. Compared to other environmental monitoring and modelling programmes the moss surveys registered increasing concentrations of toxic metal elements between 1990 and 2005, e. g. Cr. High Cr loads in mosses were also registered in other European countries like in Switzerland. Further investigations are therefore necessary to investigate whether this is due to different emission conditions or biogenic effects (e.g. as a result of increasing nitrogen depositions). Contrary to deposition measurements that exhibit a higher temporal resolution the moss surveys provide measurement data on a wide range of elements. Some of these elements are important with regard to human-toxicological aspects (e.g. As, $\mathrm{Al}, \mathrm{Hg}, \mathrm{Sb}, \mathrm{V}$ ). Due to its ecotoxicological relevance nitrogen was monitored in the European moss survey 2005 for the first time. Compared to the metals regionally high emissions of nitrogen compounds into the atmosphere can be detected in Lower Saxony.

The standardised biomonitoring of atmospheric pollution with mosses is an important link between the technical acquisition of depositions and the accumulation in biological material. To claim that the element concentrations in mosses should correlate to a high degree with measured or modelled depositions is not appropriate since these approaches are considering different biological or physical receptors. Nevertheless, the accumulation of air pollutants in terricolous mosses reflects the degree of air pollution which is permanently deposited and affects the system of plants and soils. The degree of correlation thereby depends on the boundary conditions of the physical processes, like regional and sitespecific meteorological conditions within the accumulation period, the vertical and horizontal vegetation structure or land use conditions.

Conclusions The moss surveys contribute to the heavy metal and the multi-component-model of CLRTAP because they prove on different spatial scales how air pollution control influences the accumulation of emitted substances in environmental subjects of protection like vegetation incl. arable crops. If environmental monitoring is seen as a continuous task and the applied methodology works well as an early warning system then environmental policy is enabled to act in preventative sense and to pursue unexpected developments. No other environmental monitoring programme provides such a wide range of ecotoxicologically relevant elements measured as spatially dense as the case for the moss surveys. The spatial distribution of environmental information is an essential criterion for their usability in terms of political measures for the federal states and the federation.

Recommendations and perspectives Heavy Metals in Mosses Surveys are a positive example for environmental monitoring activities reaching across three spatial and administrative levels: regional (e.g. federal state or natural landscape), nation wide (e.g. Germany) and continental (e.g. Europe). In Germany the harmonised and quality controlled moss data are made available via a WebGIS portal. Therefore the moss data may easily be accessed for environmental monitoring purposes and the control of environmental political actions. Hence, the continuous task of 
environmental monitoring can be met and carried on in the future.

Keywords Air pollution · Bioaccumulation · CART (Classification and Regression Trees) · CHAID (Chisquare Automatic Interaction Detection) · Geostatistics · Heavy metals $\cdot$ Mosses Survey $\cdot$ Nitrogen $\cdot$ Predicted environmental concentration

\section{Hintergrund, Ziel und Bereich}

Bodenbewohnende Moose können über mehrere Jahre Schwermetalle und andere Schadstoffe ohne physiologische Beeinträchtigung anreichern. Sie eigenen sich daher für ein Biomonitoring derartiger Luftverunreinigungen auf lokaler und regionaler bis hin zur kontinentalen Ebene (Bealey et al. 2008a,b; Forster et al. 1993; Genßler et al. 2001; Herpin et al. 2004; Kostka-Rick et al. 2001; Mohr 1999, 2007; Rühling und Tyler 1968, 1969, 1970; Schröder et al. 2008a, 2008b; Tylor 1990; Wappelhorst et al. 2000; Zechmeister et al. 2006). Deutschland beteiligt sich seit 1990 alle fünf Jahre an den Heavy Metals in Mosses Surveys, die von der UNECE auf Grundlage des Genfer Luftreinhalteabkommens (Convention on Long-Range Transboundary Air Pollution, CLRTAP) im Fünfjahresturnus an mindestens 1,5 Standorten pro $1000 \mathrm{~km}^{2}$ europaweit durchgeführt werden. Dabei werden die Anreicherungen von Metallen (seit 1990) und Stickstoff (seit 2005) ermittelt. Die Auswertung und Darstellung der Ergebnisse erfolgt in Berichten der Working Group on Effects (WGE) der CLRTAP und des Programmzentrums des International Cooperative Programme on Effects of Air Pollution on Natural Vegetation and Crops (ICP Vegetation) in Bangor, Wales (http://icpvegetation.ceh. ac.uk/) (Harmens et al. 2008). Die aktuellste Auswertung der Metall- und Stickstoffgehalte in Deutschland zwischen 1990 und 2005 legten Schröder et al. (2009) vor.

In Deutschland führen Bund und Länder das Moosmonitoring gemeinsam durch: Im Auftrag des Umweltbundesamtes (UBA) erfolgt die chemische Analyse der Moose und die Datenauswertung, den Bundesländern obliegt die Durchführung der Moosprobenentnahme. Der Arbeitskreis Bioindikation/Wirkungsermittlung der Landesämter und -anstalten für Umweltschutz (AKB) und ein Vertreter des UBA begleiten das Moosmonitoring als wissenschaftlicher Beirat. Folglich besteht ein Interesse, neben den europaweiten und bundesweiten Auswertungen, diese räumlich differenzierter auch auf der Ebene der Bundesländer vorzunehmen. Dieser Artikel behandelt die Kartierung der Metallanreicherung in Niedersachsen seit 1990, der Stickstoffanreicherung 2005 sowie die räumliche Varianz der Metallbioakkumulation in Abhängigkeit von Eigenschaften der Probeentnahmestellen und ihrer Umgebung.

\section{Material und Methoden}

\subsection{Datenerhebung}

Das Moosmonitoring wurde in Deutschland 1990, 1995, 2000 und 2005 durchgeführt. Dabei entfielen auf die Fläche von Niedersachsen und Bremen $\left(47.572 \mathrm{~km}^{2}\right) 82$ (1990), 130 (1995), 95 (2000) bzw. 88 (2005) Moossammelorte, was einer räumlichen Dichte von 1,7, 2,6, 2,0 bzw. 1,8 Probenorten pro Quadratkilometer entspricht und damit die Mindestvorgabe der Richtlinie (1,5 Moosprobenentnahmestandorte pro $1000 \mathrm{~km}^{2}$ ) erfüllt. Das Messnetz wurde von 2000 bis 2005 ohne Einschränkung seiner geostatistischen Validität und Landschaftsrepräsentanz ausgedünnt (Pesch et al. 2008; Schröder et al. 1991, 2004; Schröder und Schmidt 2000).

Die deutsche Moosprobenentnahme erfolgte in Anlehnung an die europaweit verwendete Richtlinie (Harmens 2005). Die Massenkonzentrationen der Elemente Aluminium (Al), Barium (Ba), Calcium (Ca), Kupfer $(\mathrm{Cu})$, Eisen $(\mathrm{Fe})$, Kalium $(\mathrm{K})$, Magnesium $(\mathrm{Mg})$, Mangan $(\mathrm{Mn})$, Natrium (Na), Strontium (Sr), Titan (Ti) und Zink (Zn) wurden in der Aufschlusslösung mit ICP-OES nach DIN EN ISO 11885 (E 22) und VDLUFA Methodenbuch 2.2.2.6 gemessen. Die Massenkonzentrationen der Elemente Arsen (As), Cadmium (Cd), Kobalt (Co), Chrom (Cr), Molybdän (Mo), Nickel (Ni), Blei (Pb), Antimon ( $\mathrm{Sb}$ ) und Vanadium (V) wurden mit ICP-MS nach DIN 38406-29 (E 29) und VDLUFA Methodenbuch 2.2.2.5 bestimmt, die Gehalte von Quecksilber ( $\mathrm{Hg})$ in den Moosen mit einem Quecksilberanalysator (thermostatisierte Zweiwegzelle) nach DIN EN 1483 und VDLUFA Methodenbuch VII. Die Massenkonzentration vom Gesamt-N (Gesamtstickstoff) wurde mit einem $\mathrm{C} / \mathrm{N}$-Analyzer (Wärmeleitfähigkeitsdetektor) nach VDLUFA Methodenbuch II 3.5.2.7 durch die Verbrennung von 0,2 g Moosprobenmaterial im Sauerstoffstrom ermittelt.

Die Qualität der Messungen und Probenentnahmen wurde, beginnend mit der Planung und der Schulung der Probenentnehmer, in mehreren Schritten sichergestellt und kontrolliert (Funk et al. 2006, Schröder et al. 1991, 2004, 2009): Die analytischen Kontrollen erfolgten wie in anderen Teilnehmerstaaten durch die Messung der Referenzmaterialien M2 (hohe Metallkonzentrationen) und M3 (Hintergrundwerte) in jeder Messserie sowie durch den Austausch und die Messung von Moosproben, die auf beiden Seiten der Grenzen benachbarter Staaten gesammelt wurden. Die Daten wurden von den Teilnehmerstaaten und nachfolgend vom Programmzentrum des ICP Vegetation daraufhin überprüft, ob sie den Anforderungen nach Steinnes et al. (1997) entsprechen. In Deutschland erfolgte dies zuvor durch den Arbeitskreis Bioindikation/Wirkungsermittlung der Landesanstalten und -ämter für Umweltschutz: Auffäl- 
lige Messwerte, die entweder über dem bundes- oder dem landesweiten 98. Perzentil lagen, wurden anhand der Metadaten im WebGIS MossMet (Abschn. 2.2) zusammen mit Geoinformationen u. a. zur Landnutzung in der Umgebung der Moosprobenentnahmeorte geprüft. Hierzu wurden die über das WebGIS MossMet zugänglichen Probenentnahmeprotokolle und Standortbeschreibungen analysiert sowie Probensammler und ortskundige Fachleute befragt. Ferner wurde ermittelt, ob die Daten derselben Messpunkte bereits bei vorangegangenen Kampagnen auffällig waren. Anschließend wurde eingeschätzt, ob die Werte der regional zu erwartenden Belastung entsprechen. Erschien der gefundene Wert auch danach nicht erklärbar, so wurde dieser nicht weiter berücksichtigt.

\subsection{Datenauswertung}

Zur räumlichen Darstellung der Belastungssituation wurden geostatistische Methoden eingesetzt (Matheron 1965; Webster und Oliver 2001). Grundlage hierfür waren sowohl die Metall- und Stickstoffkonzentrationen im Moosgewebe (in \% TS) als auch ein synoptischer Multimetallindex (MMI, s. u.). Die Qualität der dazu berechneten Flächenschätzungen wurde durch Kreuzvalidierung ermittelt (Johnston et al. 2001; Pesch 2003; Pesch et al. 2007b). Für die Berechnung des Multimetallindizes (MMI) wurden pro Element zehn Perzentilklassen gebildet, und jedem Perzentil wurde dann ein Indexwert zwischen 1 (niedrige Akkumulation) und 10 (hohe Anreicherung) zugewiesen. Der $\mathrm{MMI}_{1990-2005}$ eines jeden Moossammelortes oder einer anderen räumlichen Einheit entspricht dem Durchschnitt der dort ermittelten elementspezifischen Indexwerte der Konzentrationen von $\mathrm{As}, \mathrm{Cd}, \mathrm{Cr}, \mathrm{Cu}, \mathrm{Fe}, \mathrm{Ni}, \mathrm{Pb}, \mathrm{V}$, Ti und $\mathrm{Zn}$, die durchgängig 1990, 1995, 2000 und 2005 in Niedersachsen gemessen bzw. geostatistisch geschätzt wurden.

Alle Ergebnisse und weiterführenden Informationen der Moosmonitoringkampagnen wurden im WebGIS MossMet dokumentiert und sind dem Bund, den Ländern sowie dem ICP Vegetation zugänglich (Kleppin et al. 2008b; Pesch et al. 2007a). Die Messdaten der Monitoringkampagnen 1990, 1995, 2000 und 2005 sowie die daraus berechneten MMI konnten daher von allen Beteiligten kurzfristig überprüft sowie korrelationsstatistisch untersucht werden auf:

1. Standortspezifische Informationen (s.u.), ökologische und topografische Standorteigenschaften, Ablauf und meteorologische Randbedingungen der Moosprobenentnahme und

2. flächenhafte Informationen über das Klima, die Höhenlage über NN, Flächenanteile forstlicher, agrarischer und urbaner Landnutzung nach CORINE Land Cover (Keil et al. 2005), Entfernung zu großen Industriebetrieben sowie Immissionen in der Umgebung der Moosmonitoringstandorte.
Hierbei handelt es sich um Daten über atmosphärische Depositionen aus dem EMEP-Luftmessnetz des Umweltbundesamtes (wet only), aus dem ICP-Forest-Messnetz (Freiland- und Bestandsdeposition) sowie Flächenschätzungen der modellierten Nass-, Trocken- und Gesamtdeposition. Stärke und Richtung der statistischen Zusammenhänge zwischen

1. jeweils zwei Metallgehalten in den Moosen,

2. zwischen Metallkonzentrationen in Moosen und gemessenen Depositionen (bulk und wet only Freiland, Bestandsdeposition) und

3. zwischen Metallgehalten und modellierter Nass-, Trocken- und Gesamtdeposition

wurden durch Rangkorrelationskoeffizienten nach Spearman $r_{\mathrm{s}}$ bzw. durch das Assoziationsmaß Cramér's V quantifiziert. Zur näheren Eingrenzung möglicher Belastungsursachen wurden zusätzlich multivariat-statistische Korrelationsmuster mit Classification and Regression Trees (CART, Breimann et al. 1984) berechnet. Dabei bildeten die Gehalte der in den Moosen 2005 gemessenen Stoffgehalte jeweils die Zielvariable. Als Prädiktoren dienten die standortbeschreibenden Metainformationen sowie flächenhaft vorliegende Daten zur Umgebungsbeschreibung.

An kleinflächigen Sammelorten zwischen Gehölzen werden die Moosproben oftmals stärker mit reaktivem Stickstoff (Ammonium, Nitrat) beaufschlagt, wodurch es $\mathrm{zu}$ einer Verzerrung des großräumigen Verteilungsmusters kommt (Mohr 2007). Um diesen in manchen Regionen unvermeidbaren Einfluss zu berücksichtigen, erfolgte eine Umrechnung der Stickstoffkonzentrationen mit den Schwefelgehalten in den Moosen. Dieses Vorgehen begründet sich aus der Feststellung, dass die S-Konzentrationen in Moosen bei den aktuell sehr niedrigen $\mathrm{SO}_{2}$-Immissionen großräumig nur gering variieren (Mohr 1999). Unterschiede der S-Gehalte sind somit in großen Teilen Deutschlands vorrangig auf abweichende Depositionsbedingungen am Probenentnahmeort zurückzuführen, die in großen Teilen Niedersachsens durch die unterschiedliche Vegetationsstruktur hervorgerufen werden. Aufgrund der hohen Korrelation sedimentierender S- und N-Einträge im Niederschlag (Dämmgen 2005) sowie solcher in den untersuchten Moospflanzen (2005: $r=0,83 ; p<0,0001)$ kann der S-Gehalt als Indikator für den Traufeeinfluss der Begleitvegetation (vornehmlich Bäume und Sträucher) und zur Standardisierung der N-Gehalte herangezogen werden. Die Umrechnung basiert auf der folgenden empirischen Formel: $\mathrm{N}_{\text {korrigiert }}=[1000 / \mathrm{S}-$ Konzentration $] \times \mathrm{N}_{\text {gemessen }}$. Hierbei entspricht $1000 \mu \mathrm{gg}^{-1} \mathrm{~S}$ der zu erwartenden S-Bioakkumulation unter Freilandbedingungen. Dieser Wert wurde landesweit unter optimalen Freilandbedingungen festgestellt, höhere S-Konzentrationen resultierten aus Probenentnahmen in nicht ausreichender Entfernung $(<10 \mathrm{~m}) \mathrm{zu}$ Gehölzbeständen. 
Tabelle 1 Beprobte Moosarten in Niedersachsen 1990 bis 2005

\begin{tabular}{rrrrrrrrr}
\hline & $\begin{array}{c}\text { Brachytecium } \\
\text { rutabularum }\end{array}$ & \multicolumn{2}{c}{$\begin{array}{c}\text { Hypnum } \\
\text { cupressiforme }\end{array}$} & \multicolumn{2}{c}{$\begin{array}{c}\text { Pleurozium } \\
\text { schreberi }\end{array}$} & \multicolumn{2}{c}{$\begin{array}{c}\text { Scleropodium } \\
\text { purum }\end{array}$} \\
\hline & $n$ & $\%$ & $n$ & $\%$ & $n$ & $\%$ & $n$ & $\%$ \\
1990 & 0 & 0,0 & 4 & 4,9 & 72 & 87,8 & 6 & 7,3 \\
1995 & 9 & 6,9 & 6 & 4,6 & 88 & 67,7 & 27 & 20,8 \\
2000 & 1 & 1,1 & 9 & 9,5 & 60 & 63,2 & 25 & 26,3 \\
2005 & 0 & 0,0 & 12 & 13,6 & 48 & 54,5 & 28 & 31,8 \\
\hline
\end{tabular}

\section{Ergebnisse}

Von 1990 bis 2005 ist Pleurozium schreberi das am häufigsten in Niedersachsen gesammelte Moos, gefolgt von Scleropodium purum und Hypnum cupressiforme (Tabelle 1). In Niedersachsen liegt der Anteil von Pleurozium schreberi an den gesammelten Moosproben um gut 10\% über dem Bundesdurchschnitt im Jahr 2005 (42,2\%). Scleropodium purum hat in Niedersachsen 2005 fast denselben Anteil wie im Bund (hier: 32,6\%), der Anteil von Hypnum cupressiforme unterschreitet den Wert im Bund um gut $10 \%$.

Abbildung 1 stellt beispielhaft die räumliche Verteilung und die Entwicklung der Cd-Gehalte in den Moosproben innerhalb Niedersachsens dar. Viele der seit 1990 analysierten Metalle wiesen ebenfalls eine deutlich rückläufige Tendenz auf. Besonders ausgeprägt war z.B. der Rückgang des seit 1996 in Kraftstoffen nicht mehr enthaltenen Bleis.

Tabelle 2 gibt anhand der Perzentilstatistik und der zusammenfassenden MMI einen zusammenfassenden Überblick der zeitlichen Entwicklung der Bioakkumulation der zwölf Standardmetalle. Anhand des 20., 50. und 90. Perzentils sowie des MMI lässt sich eine allgemein deutliche, zumeist kontinuierliche Abnahme der Metallanreicherung in Moosen zwischen 1990 und 2005 nachweisen. Das 50. Perzentil des MMI sank von 7,15 im Jahr 1990 deutlich auf 3,4 im Jahr 2000. 2005 stieg dieser Wert allerdings signifikant auf 4,0. Signifikante Verminderungen wurden für die $\mathrm{Cd}-, \mathrm{Pb}-$ und

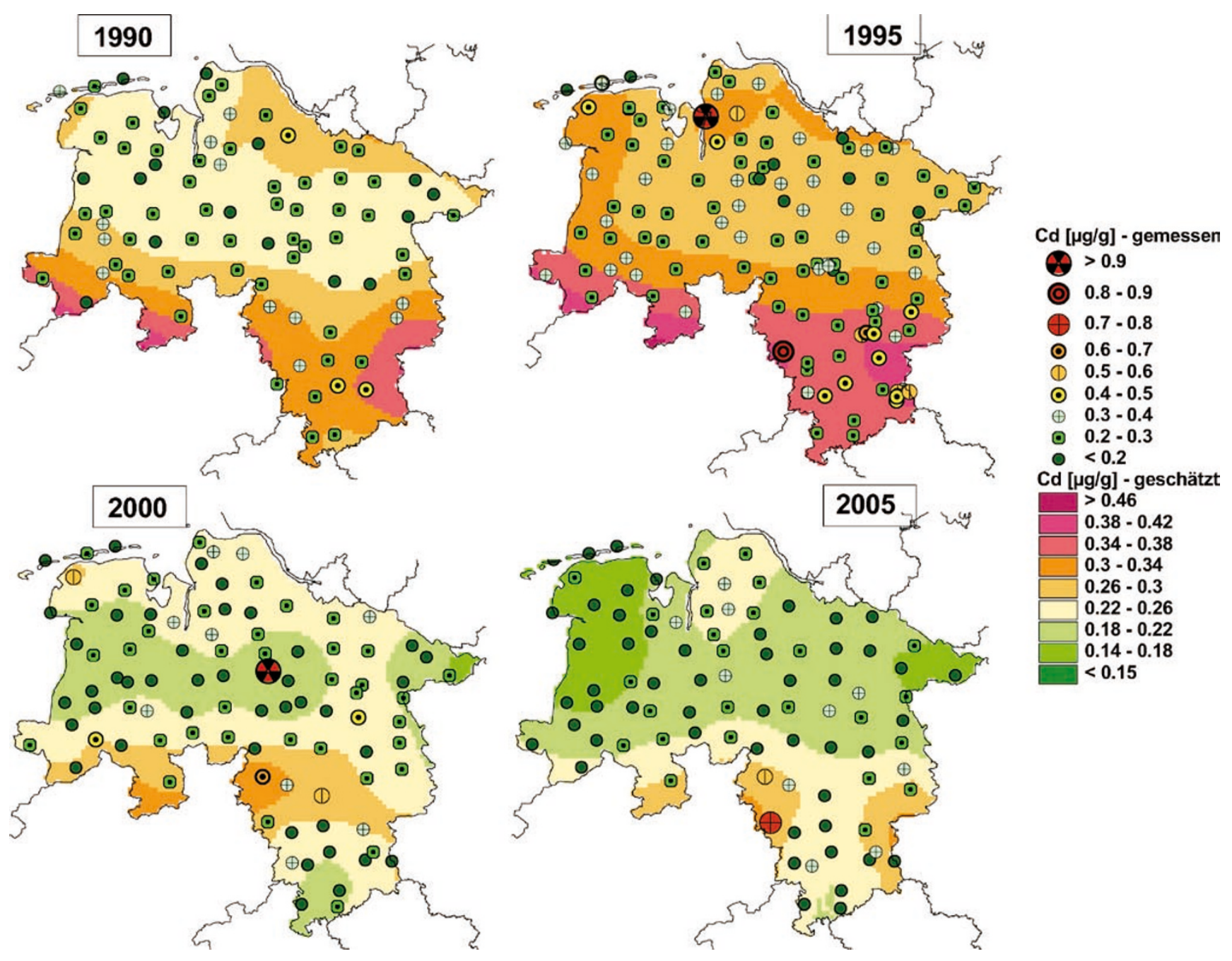

Abb. 1 Cd-Gehalte in niedersächsischen Moosen 1990 bis 2005 
Tabelle 2 Deskriptiv-statistische Maßzahlen der Elementgehalte in niedersächsischen Moosen 1990 bis 2005

\begin{tabular}{|c|c|c|c|c|c|c|c|c|c|c|c|c|c|}
\hline \multicolumn{14}{|c|}{1990} \\
\hline & As & Cd & $\mathrm{Cr}$ & $\mathrm{Cu}$ & $\mathrm{Fe}$ & Hg & $\mathbf{N i}$ & $\mathbf{P b}$ & Sb & $\mathbf{T i}$ & $\mathbf{V}$ & Zn & MMI \\
\hline Anzahl & 82 & 82 & 82 & 82 & 82 & 0 & 82 & 82 & 0 & 82 & 82 & 82 & 82 \\
\hline 20. Perz. & 0,156 & 0,200 & 1,41 & 5,51 & 340 & & 1,26 & 9,93 & & 20,1 & 2,68 & 30,8 & 6 \\
\hline 50. Perz. & 0,271 & 0,255 & 1,58 & 6,47 & 416 & & 1,66 & 11,50 & & 23,4 & 3,19 & 36,1 & 7,15 \\
\hline 90. Perz. & 0,428 & 0,339 & 2,67 & 8,98 & 666 & & 2,62 & 16,04 & & 32,5 & 4,39 & 51,0 & 8,87 \\
\hline
\end{tabular}

1995

\begin{tabular}{|c|c|c|c|c|c|c|c|c|c|c|c|c|c|}
\hline & As & Cd & $\mathrm{Cr}$ & $\mathrm{Cu}$ & $\mathbf{F e}$ & Hg & $\mathbf{N i}$ & $\mathbf{P b}$ & Sb & $\mathbf{T i}$ & $\mathbf{V}$ & Zn & MMI \\
\hline Anzahl & 130 & 130 & 130 & 130 & 130 & 130 & 130 & 130 & 130 & 130 & 130 & 130 & 130 \\
\hline 20. Perz. & 0,100 & 0,230 & 1,09 & 6,14 & 288 & 0,020 & 1,07 & 6,25 & 0,130 & 12,6 & 1,48 & 40,6 & 5,52 \\
\hline 50. Perz. & 0,190 & 0,290 & 1,45 & 7,23 & 378 & 0,030 & 1,41 & 8,15 & 0,190 & 17,0 & 2,21 & 51,4 & 6,6 \\
\hline 90. Perz. & 0,377 & 0,439 & 2,75 & 9,72 & 767 & 0,059 & 2,52 & 13,78 & 0,280 & 31,8 & 3,80 & 74,6 & 8,99 \\
\hline
\end{tabular}

\begin{tabular}{|c|c|c|c|c|c|c|c|c|c|c|c|c|c|}
\hline \multicolumn{14}{|c|}{2000} \\
\hline & As & Cd & $\mathrm{Cr}$ & $\mathrm{Cu}$ & $\mathrm{Fe}$ & Hg & $\mathbf{N i}$ & $\mathbf{P b}$ & Sb & $\mathbf{T i}$ & $\mathbf{V}$ & Zn & MMI \\
\hline Anzahl & 95 & 95 & 95 & 95 & 95 & 95 & 95 & 95 & 95 & 95 & 95 & 95 & 95 \\
\hline 20. Perz. & 0,080 & 0,160 & 0,64 & 4,65 & 191 & 0,025 & 0,70 & 2,99 & 0,102 & 3,5 & 0,81 & 30,9 & 2,5 \\
\hline 50. Perz. & 0,120 & 0,200 & 0,83 & 5,57 & 246 & 0,031 & 0,87 & 4,14 & 0,150 & 4,9 & 1,19 & 38,2 & 3,4 \\
\hline 90. Perz. & 0,184 & 0,334 & 1,21 & 7,53 & 432 & 0,046 & 1,24 & 6,05 & 0,252 & 9,0 & 1,71 & 53,4 & 5,44 \\
\hline
\end{tabular}

2005

\begin{tabular}{|c|c|c|c|c|c|c|c|c|c|c|c|c|c|}
\hline & As & Cd & $\mathrm{Cr}$ & $\mathrm{Cu}$ & $\mathrm{Fe}$ & Hg & $\mathrm{Ni}$ & $\mathbf{P b}$ & Sb & $\mathbf{T i}$ & V & $\mathrm{Zn}$ & MMI \\
\hline Anzahl & 88 & 88 & 88 & 88 & 88 & 88 & 88 & 88 & 88 & 88 & 88 & 88 & 88 \\
\hline 20. Perz. & 0,089 & 0,120 & 1,42 & 4,93 & 192 & 0,023 & 0,65 & 2,31 & 0,100 & 3,2 & 0,89 & 31,1 & 2,9 \\
\hline 50. Perz. & 0,120 & 0,180 & 2,14 & 5,78 & 247 & 0,029 & 0,86 & 3,27 & 0,160 & 4,6 & 1,18 & 40,3 & 4 \\
\hline 90. Perz. & 0,181 & 0,321 & 5,34 & 7,91 & 398 & 0,040 & 1,37 & 5,83 & 0,235 & 7,9 & 1,80 & 66,9 & 6,31 \\
\hline
\end{tabular}

Hg-Werte im Vergleich der Messkampagnen 2000 und 2005 nachgewiesen. Andere Unterschiede der Werte zwischen den Surveys 2000 und 2005 erwiesen sich nicht als signifikant. Eine Ausnahme bildet Cr, dass 2005 gegenüber 2000, 1995 und 1990 signifikant anstieg (Tabelle 3). Vergleichbare Auffälligkeiten der Cr-Gehalte, die 2005 mit Werten über $5 \mathrm{mg} \mathrm{kg}^{-1}$ deutlich über dem bundesweiten Durchschnittswert $\left(3,71 \mathrm{mg} \mathrm{kg}^{-1)}\right.$ lagen, wurden auch in anderen Bundesländern festgestellt (Schröder et al. 2009). Da die Probenentnahmeorte sich in großer Entfernung (z.B. auf den Ostfriesischen Inseln) zu potenziellen Emittenten befanden und auch vorhandene Depositionsmessdaten keine Hinweise zu möglichen Immissionseinflüssen gaben, erfolgte im Jahr 2007 eine wiederholte Beprobung an ausgewählten Punkten.

Die in Tabelle 4 aufgeführten Ergebnisse zeigen, dass die hohen Cr-Konzentrationen der 2006 belasteten Moosproben
Tabelle 3 Inferenz-statistische Analyse der Entwicklung der Stoffanreicherungen in Niedersachsen 1990 bis 2005
As $\begin{array}{lllllllllllll} & \mathrm{Cd} & \mathrm{Cr} & \mathrm{Cu} & \mathrm{Fe} & \mathrm{Hg} & \mathrm{Ni} & \mathrm{Pb} & \mathrm{Sb} & \mathrm{Ti} & \mathrm{V} & \mathrm{Zn} & \mathrm{MMI}\end{array}$

\begin{tabular}{|c|c|c|c|c|c|c|c|c|c|c|c|c|c|}
\hline 1990 - 1995 & A & $\mathbf{Z}$ & A & $\mathbf{Z}$ & A & & A & A & & A & A & $\mathbf{Z}$ & A \\
\hline $1990-2000$ & A & A & A & A & A & & A & A & & A & A & $\mathbf{Z}$ & A \\
\hline $1990-2005$ & $\mathrm{~A}$ & A & $\mathbf{Z}$ & A & A & & A & A & & A & A & $\mathbf{Z}$ & A \\
\hline $1995-2000$ & A & A & A & A & A & $\mathbf{Z}$ & A & A & A & A & A & A & A \\
\hline $1995-2005$ & A & A & $\mathbf{Z}$ & A & A & A & A & A & A & A & A & A & A \\
\hline $2000-2005$ & A & A & $\mathbf{Z}$ & $\mathbf{Z}$ & $\mathbf{Z}$ & A & A & A & $\mathbf{Z}$ & A & A & $\mathbf{Z}$ & $\mathbf{Z}$ \\
\hline
\end{tabular}

Signifikante Änderung ( $A=$ Abnahme; $Z=$ Zunahme; $p<0,01)$

Signifikante Änderung ( $A=$ Abnahme; $Z=Z$ Zunahme; $0,01<p<0,05$ )

keine signifikante $\mathrm{Zu}$ - oder Abnahme 
Tabelle 4 Ergebnisse der Wiederholungsbeprobung an belasteten und unbelasteten Probenentnahmeorten

\begin{tabular}{|c|c|c|}
\hline & $\begin{array}{l}\text { Beprobung } \\
\text { MM } 2005\end{array}$ & $\begin{array}{c}\text { Nachbeprobung } \\
2007\end{array}$ \\
\hline Sammelort & $\mathrm{Cr}$ [mg kg-1 TS] & \\
\hline NI 31 & 1,8 & 1,6 \\
\hline NI 96 & 2,4 & 1,6 \\
\hline NI 289 & 9 & 3,2 \\
\hline NI 74 & 9,6 & 4,5 \\
\hline NI 16 & 20,1 & 5,6 \\
\hline
\end{tabular}

(NI 16, NI 74, NI 289) sich - auf einem niedrigeren Niveau - in der Tendenz bestätigten. Wesentliche Unterschiede bei Proben, die an vergleichsweise unbelasteten Standorten (NI 31, NI 96) genommen wurden, zeigten sich nicht. Dieser Befund deckt sich mit den Ergebnissen der Wiederholungsbeprobung in den Bundesländern Mecklenburg-Vorpommern und Nordrhein-Westfalen. Eine Ursache hierfür ließ sich noch nicht abschließend erkennen. Häufig wiesen die Cr-belasteten Proben in den norddeutschen Gebieten einen starken Besatz mit Grünalgen auf, was auf das humide Klima und die in dieser Region erhöhten N-Depositionen zurückzuführen ist (Mohr 2007). Grünalgen weisen eine hohes Potenzial zur Biosorption von Schwermetallen auf (Klimmek 2003).Untersuchungen der aus coccalen und fädigen Algen bestehenden Gallerte ergaben zum Teil sehr hohe Cr-Konzentrationen zwischen 2 und $38 \mathrm{mg} \mathrm{kg}^{-1}$.

Die räumliche Differenzierung der in Tabelle 2 zusammenfassend beschriebenen zeitlichen Entwicklung der Stoffanreicherung wird am Beispiel des $\mathrm{MMI}_{1990-2005}$ in Abb. 2 und für Stickstoff in Abb. 3 kartografisch veranschaulicht. Die noch 1990 und 1995 von den industriellen Regionen im Südosten Niedersachsens und von benachbarten Bundesländern geprägten Unterschiede der Schwermetallbelastung nivellierten sich in den vergangenen 15 Jahren weitgehend. Anhand niedriger MMI-Werte wird im Küstenraum die niedrigste Hintergrundbelastung ersichtlich. Ein moderater Anstieg im Küstenraum, zwischen 1990 und 1995 war im wesentlich durch die Elemente $\mathrm{Cd}, \mathrm{Cu}$, und $\mathrm{Zn}$ bedingt. Die Belastungen der Elbregionen im Osten Niedersachsens fielen schon in den ersten Jahren nach der Wende auf das niedrige Niveau der der Küstengebiete und des westniedersächsischen Raumes. Diese Entwicklung setzte sich in den drauffolgenden Jahren in großen Teilen Niedersachsens fort. Erhöhte Schwermetallbelastungen in den Jahren 2000 und 2005 ließen sich nur noch in den südlichen Randgebieten
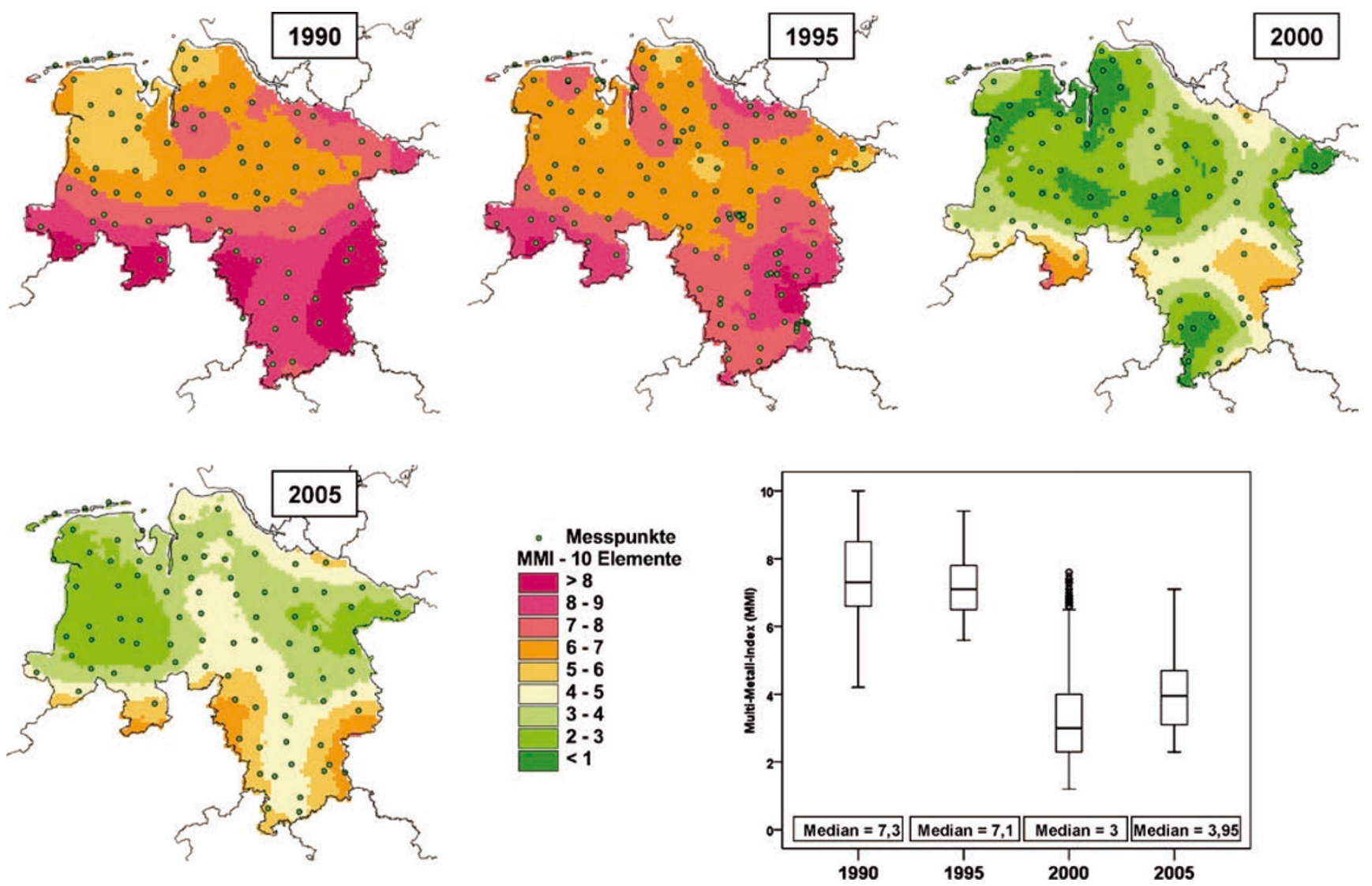

Abb. 2 Räumliches Muster des Multimetallindex MMI (As, Cd, Cr, Cu, Fe, Ni, Pb, Ti, V und Zn) in Niedersachsen 1990 bis 2005 

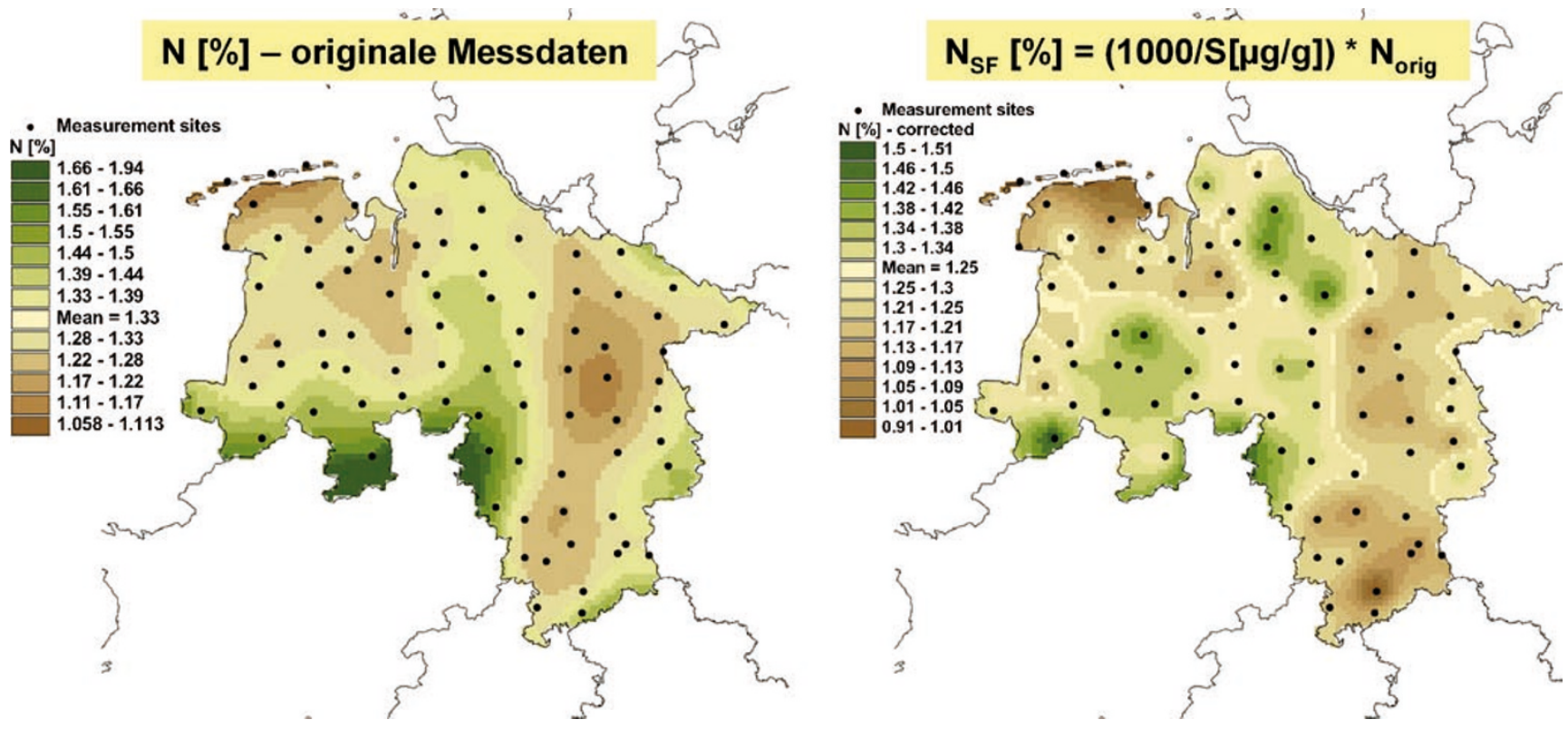

Abb. 3 Stickstoffakkumulation in Niedersachsen 2005

zu Nordrhein-Westfalen und Sachsen-Anhalt feststellen. In den (emittentenfernen) Gebieten der Industrieregion Salzgitter unterschieden sich im Jahr 2005 die MMI-Werte nur unwesentlich von denen anderer Regionen.

Das räumliche Muster der Stickstoffanreicherung wird anhand der Originalmessdaten sowie anhand der mit Schwefel korrigierten Werte (Abschn. 2.2) dargestellt. Beide Auswertungen weisen auf relativ hohe Stickstoffbelastungen in den westlichen Landesteilen hin. Die niedrigsten N-Konzentrationen im Moos wurden großräumig im östlichen und südlichen Niedersachsen festgestellt. Deutlicher werden die Unterschiede anhand der schwefelkorrigierten Werte. Hierdurch heben sich die landwirtschaftlich intensiv genutzten Regionen Südoldenburgs, des Münsterlandes und des Elbe-Weser-Dreiecks hervor. Weniger durch intensive Tierhaltung geprägte Regionen der ostfriesischen Inseln, des Leine-Weserberglandes, des Harzes und der Lüneburger-Heide wiesen die niedrigsten Werte auf.

Bei der Untersuchung von statistischen Zusammenhängen zwischen der Metallanreicherung und möglichen Einflüssen konnten die Daten zu atmosphärischen Depositionen nicht herangezogen werden, da diese für Niedersachsen nicht in ausreichender Zahl verfügbar waren. Jedoch wurden die bei der Moosprobenentnahme erhobenen Informationen über Eigenschaften der Sammelorte berücksichtigt, welche die Landnutzung ihrer Umgebung und insofern potenzielle Quellen für Metalle und Stickstoff kennzeichnen. Die Ergebnisse der bivariaten Zusammenhangsanalysen werden für metrische Daten in Tabelle 5 in Form des Rangkorrelationskoeffizienten nach Spearman $\left(r_{\mathrm{s}}\right)$ und in Tabelle 6 für die kategorialen Daten durch Cramér's $V$-Werte präsentiert.
Die Werte dieser beiden Zusammenhangsmaße liegen, sofern statistisch signifikant, überwiegend zwischen 0,2 und 0,5. Werte zwischen 0,5 und 0,65 finden sich beim Zusammenhang zwischen Moosart und der Anreicherung von Ni, $\mathrm{Pb}$ und $\mathrm{Sb}$ sowie zwischen der Moosbewuchsform (rasen-, polsterförmig) und den Cd-Gehalten. Negativ korreliert sind die Stoffgehalte in Moosen mit der Nähe zu Bäumen und Sträuchern. Derartige Vegetationsstrukturen filtern die Stoffe aus der Luft aus und tragen so zu einer verstärkten Deposition auch auf die Bodenmoose bei. Je geringer der Anteil agrarisch genutzter Flächen im Umkreis der Beprobungsstellen, desto höher sind die Gehalte der Metalle vorwiegend industriellen Ursprungs: Negative signifikante Korrelationen mit der Agrardichte lassen sich in einigen Untersuchungsperioden v. a. für $\mathrm{As}, \mathrm{Cd}, \mathrm{Cr}, \mathrm{Hg}, \mathrm{Ni}, \mathrm{Pb}, \mathrm{Sb}$ und Ti festhalten (Tabelle 5).

Ein signifikanter Zusammenhang zu benachbarten Industrieansiedlungen gibt sich aufgrund der emittentenfernen Lage der Probenentnahmeorte nicht zu erkennen. Die Stickstoffgehalte in den Moosen weisen aus gleichem Grund keine enge räumliche Beziehung zu lokalen N-Emittenten aus Landwirtschaft, Straßenverkehr oder Industrie auf. Demgegenüber spiegelt das kartografische Verteilungsmuster die großräumige Hintergrundbelastung durch Metalle und N-haltige Immissionen sehr deutlich wider. Negativ korreliert sind die Gehalte von $\mathrm{Cr}, \mathrm{Fe}$, und Ti mit den jährlichen Niederschlagssummen sowie die TiKonzentrationen mit der Entfernung der Moosprobenentnahmestellen zu vegetationsfreien Flächen, welches auf eine überproportional hohe trockene Deposition dieser Metalle hindeutet. 
Tabelle 5 Rankkorrelation $\left(r_{\mathrm{s}}\right)$ zwischen Metallanreicherungen und Einflussfaktoren in Niedersachsen 1990 bis 2005
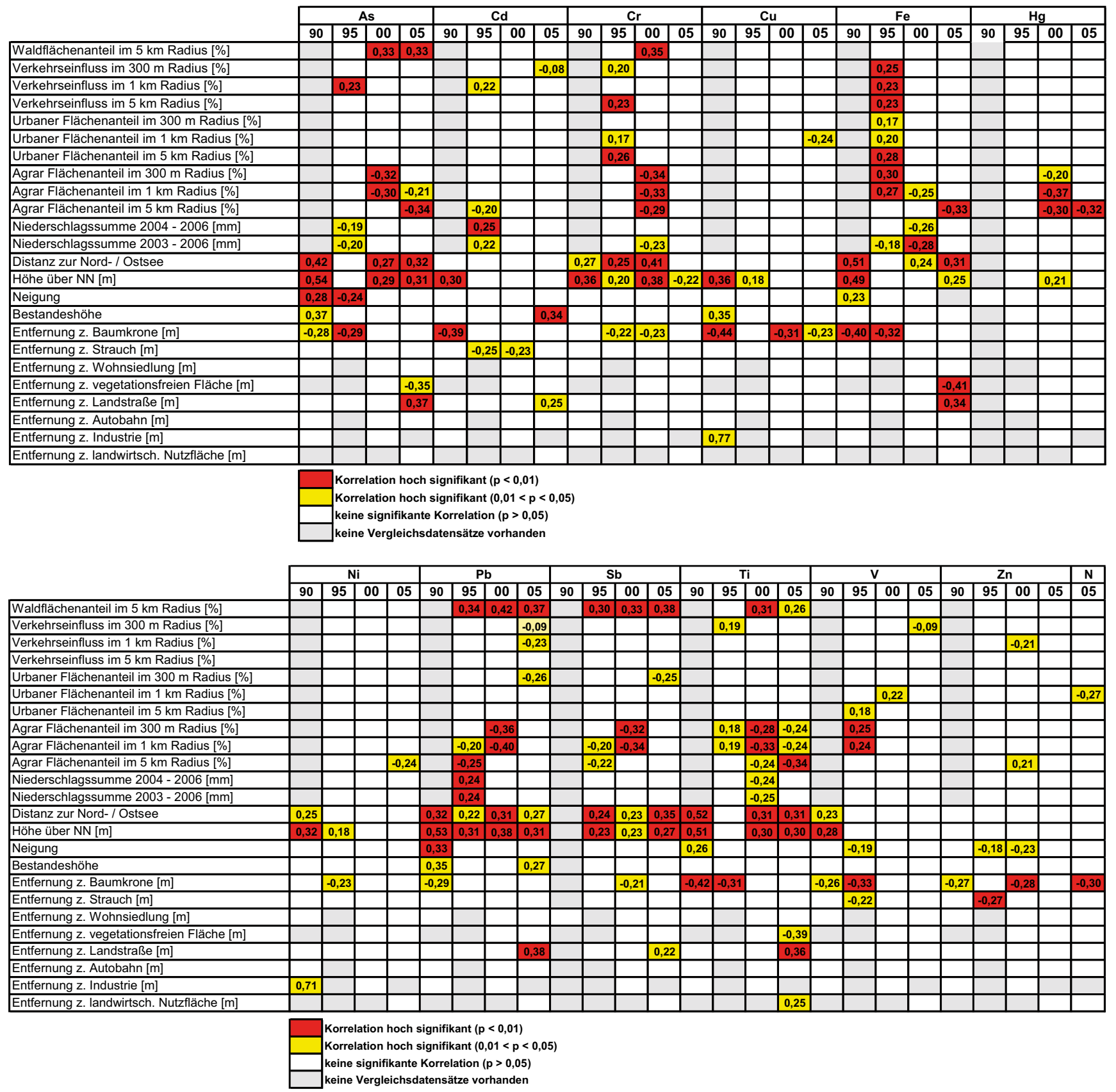

Der multivariat-statistische Zusammenhang der Metallgehalte mit Begleitfaktoren wird in Abb. 4 am Beispiel von $\mathrm{Sb}$ aufgezeigt. Das Dendrogramm zeigt die statistisch wichtigsten Prädiktoren der Sb-Konzentrationen und gegenseitige Verflechtungen. Der Knoten 0 beschreibt die Verteilung der Sb-Messwerte an den 88 im Jahr 2005 beprobten Standorten; der Mittelwert beträgt rund $0,149 \mu \mathrm{g} / \mathrm{g}$. Bezogen auf die Daten des Surveys 2005 ist die Moosart derjenige Faktor, der mit den Sb-Konzentrationen in den Moosen am engsten verknüpft ist (Cramér's $V=0,65$, Tabelle 6): Für die 28 Orte, an denen Scleropodium purum gesammelt wurde, ergibt sich eine mittlere Sb-Konzentration von $0,093 \mu \mathrm{g} / \mathrm{g}$. Dieser Wert liegt unterhalb des Mittelwertes der Untersuchungsstichprobe. Dieser wird mit $0,186 \mu \mathrm{g} / \mathrm{g}$ an denjenigen Standorten überschritten, an denen 2005 Pleurozium schreberi und Hypnum cupressiforme gesammelt wurde. Der Stichprobenmittelwert wird weiterhin übertroffen, wenn der Waldflächenanteil in einem Umkreis von $5 \mathrm{~km}$ um diese Standorte über 6\% liegt. Auch an Standorten mit Scleropodium purum sind höhere Sb-Gehalte mit höheren Waldanteilen im Umkreis von $10 \mathrm{~km}$ um die Moosbeprobungsorte verknüpft. Weitere Umgebungsmerkmale, die mit den Sb- 
Tabelle 6 Zusammenhang (Cramér's $V$ ) zwischen Metallanreicherungen und moosspezifschen Merkmalen 1990 bis 2005

Abb. 4

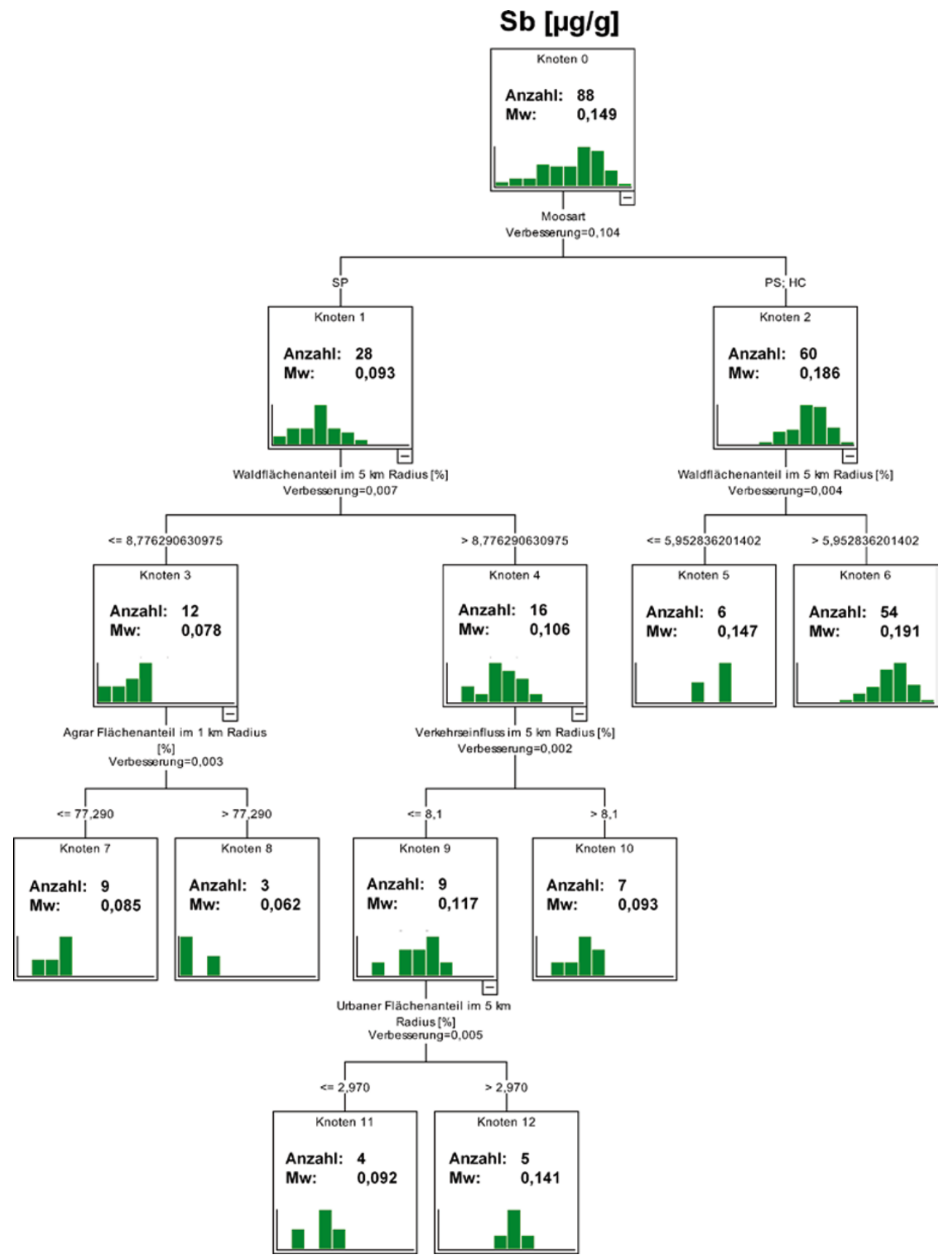


Werten schwach korrelieren, sind die Flächenanteile agrarischer und urbaner Nutzungen sowie der Verkehrsfläche in der Umgebung der Moosprobenentnahmestellen.

\section{Diskussion}

Für Niedersachsen lässt sich mit dem Moosmonitoring aus den Jahren 1990 bis 2005 nachweisen, dass die abnehmende Metallemissionen und -depositionen in Deutschland auch zu sinkenden Metallkonzentrationen in Moosen führten. Die Ergebnisse korrespondieren mit deutschlandweiten Resultaten, die von Schröder et al. (2009) zusammengefasst und hier als Diskussionsgrundlage ebenfalls herangezogen werden. Anders als bei der Erfassung der atmosphärischen Deposition (Ilyin 2009) belegt das Moosmonitoring jedoch, dass die Gehalte einiger Metalle von 2000 bis 2005 statistisch signifikant angestiegen sind. Wie im gesamten Bundesgebiet war dieser Trend in Niedersachsen bei Cr besonders deutlich, hier jedoch bereits ab 1995 und nicht erst ab 2000.

Die Korrelationsanalyse zeigt, dass die statistischen Beziehungen zwischen den in den Moosen akkumulierten Metallen und Stickstoff mit den Eigenschaften der Beprobungsorte und ihrer Umgebung plausibel und überwiegend niedrig ausgeprägt sind. Diese Zusammenhänge vermögen es, kleinräumige Varianzen der Metall- und Stickstoffgehalte zu erklären. Sie lassen sich jedoch nur für wenige Orte und Stoffe mit Daten aus Depositionsmessnetzen unterfüttern: Bulk-Depositionsdaten stehen im gesamten Bundesgebiet für Freiland und Waldbestand im Falle von Cd von $n$ $=18$ Standorten, bei $\mathrm{Cu}$ von $n=17$, bei $\mathrm{Pb}$ von $n=19$ und bei $\mathrm{Zn}$ von $n=27$ Standorten zur Verfügung. Wet-only-Depositionsdaten (Freiland) stehen für $\mathrm{As}, \mathrm{Cd}, \mathrm{Cr}$ und $\mathrm{Zn}$ von sechs Standorten des UBA-Luftmessnetzes und des ICP Integrated Monitoring zur Verfügung (Schröder et al. 2009).

Die Rangkorrelationen zwischen den Metallkonzentrationen in Moosen einerseits und den verfügbaren Depositionsdaten sowie modellierter Nass-, Trocken- und Gesamtdeposition andererseits variieren nach Stoffen und Depositionspfad. Für Niedersachsen ließ sich keine depositionsbezogene Auswertung durchführen; bundesweit geben sich - unter Berücksichtigung der niedrigen Anzahl von Vergleichsmessungen - hohe und sehr hohe Korrelationen zwischen den Konzentrationen von As und $\mathrm{Cr}$ in Moosen und in der Wet-only-Deposition zu erkennen (Schröder et al. 2009). Mittlere Korrelationen weisen die Konzentrationen von $\mathrm{Cd}, \mathrm{Cu}, \mathrm{Pb}$, and $\mathrm{Zn}$ in Moosen und Depositionen auf. Die statistischen Beziehungen zwischen den Elementkonzentrationen im Bestandesniederschlag von Wäldern und in Moosen sind bei $\mathrm{Cd}, \mathrm{Cu}$ and $\mathrm{Pb}$ gering, mittelstark hingegen für $\mathrm{Zn}$. Die Korrelationen von $\mathrm{Cd}$ and $\mathrm{Pb}$ in Moosen und den modellierten Depositionen sind niedrig bis mittel und variieren zeitlich, stoffspezifisch und nach modelliertem Depositionspfad: Die höchsten Korrelationen bestehen zwischen den Konzentrationen von $\mathrm{Cd}$ und $\mathrm{Pb}$ in Moosen und in der Gesamt- und Trockendeposition dieser Schwermetalle (Schröder et al. 2009). Für EMEP-Depositionsdaten lassen sich diese Tendenzen europaweit bestätigen: $\mathrm{Cd}$ in Depositionen und Moosen weisen eine Korrelation von $r_{\mathrm{s}}=0,65$ auf, bei $\mathrm{Pb}$ beträgt sie $r_{\mathrm{s}}=0,73$ (Holy et al. 2009c). Der Eintrag von $\mathrm{Cr}, \mathrm{Fe}$ und $\mathrm{Ti}$ erfolgt offensichtlich überproportional über die trockene Deposition. Bei Cr zeigt sich, dass durch ein Zusammenwirken dieser und anderer Umweltbedingungen möglicherweise dessen Bioverfügbarkeit erhöht wird. Die Cr-belasteten Moosproben waren häufig stark veralgt. Der durch erhöhte N-Depositionen zunehmend auch an anderen Umweltmedien festgestellte Algenbesatz (Mohr 2007) könnte dabei eine Rolle spielen. Klimmek (2003) stellte bei Zugabe von $\mathrm{Cr}^{3+}$ in Grünalgenkulturen eine rasche Oxidation zum toxischen $\mathrm{Cr}^{6+}$ fest. Der Nachweis von Chrom gilt im Umweltmonitoring sowohl bei Depositionsverfahren als auch im Biomonitoring als problematisch. Möglicherweise wird der nicht-sedimentirende Anteil der Cr-Depositionen (Feinstaub) durch die Ergebnisse des Moosmonitorings stärker hervorgehoben als der Anteil sedimentierender CrEinträge.

Die in den Moosproben in Niedersachsen erstmals ermittelten Stickstoffkonzentrationen reflektieren die regional sehr unterschiedliche Immissionsbelastung mit reaktivem Stickstoff $\left(\mathrm{NO}_{\mathrm{x}}, \mathrm{NH}_{\mathrm{y}}\right)$. Von relativ hohen N-Immissionen in Niedersachsen betroffen sind ausnahmslos ländliche Gebiete, wodurch landwirtschaftliche Quellen (insbesondere die Tierhaltung) als Hauptursache infrage kommen. Vergleichbar räumliche Differenzierungen wurden von Mohr (1999, 2007) für Niedersachsen erzielt. Im bundesweiten Vergleich liegt das Niveau der N-Konzentrationen jedoch auf niedrigem Niveau.

Der festgestellte Einfluss der Vegetation auf die Höhe der N-Deposition wird auch durch Messungen in verschiedenen Bestandesstrukturen deutlich (Dämmgen 2005). Auch im Freiland kann dieser bei nicht ausreichendem Abstand zu Gehölzen zum tragen kommen, welches die Vergleichbarkeit der Probenahmeorte beeinträchtigt. Durch die Schwefelkalibrierung der Stickstoffkonzentrationen in den Moosen ließen sich derartige Störgrößen jedoch weitgehend eliminieren, wodurch sich ein großräumig plausibles Verteilungsmuster darstellt. Durch die Probenentahme innerhalb von Wäldern (unter älterem Nadelholz) ließen sich derartige Standorteffekte ebenfalls reduzieren (Mohr 1999, 2007). Die N-Konzentrationen liegen dabei - analog zu den N-Einträgen - auf deutlich höherem Niveau.

Inwieweit Eigenschaften der Moosprobenentnahmeorte und ihrer Umgebung mit den Stoffgehalten in den Moosen statistisch verknüpft sind, wurde multivariat-statistisch untersucht und die statistische Relevanz der einzelnen Fakto- 
ren hierarchisiert. Die engsten Beziehungen bestehen zu den Moosarten, der Entfernung zu Gehölzen an der Probenentnahmestelle sowie zu der Landnutzung in ihrer Umgebung. Am Beispiel von Sb konnten die Moosart sowie Landnutzungen im Umkreis der Moossammelstellen als wichtige Faktoren ermittelt werden. Für Deutschland wurden entsprechende Untersuchungen anhand der Daten aus dem Moosmonitoring 2000 bereits von Kleppin et al. (2008a), Pesch und Schröder (2006) sowie Schröder und Pesch (2005) vorgestellt, für Frankreich von Holy et al. (2009b), für mehrere europäische Länder anhand der Monitoringdaten 2000 von Schröder et al. (2008) sowie für alle Teilnehmerstaaten des Moosmonitoring anhand der Messkampagne 2005 von Holy et al. (2009a).

Diese Untersuchungen sowie die vorliegende Studie zeigen, dass die Verknüpfung der Messdaten mit den Eigenschaften der Moossammelorte sowie deren Umgebung plausible Erklärungen für die lokale und regionale Messwertvarianz liefern. Die Aussagekraft dieser Erklärungen ist gegenüber den bis zum Jahr 2008 veröffentlichten Auswertungen gestiegen, seitdem die Informationen über die Emissionen verfügbar wurden und sich in die Auswertungen erstmals einbeziehen ließen (Holy et al. 2009a). Wenn dies auch mit den europaweiten Modellierungen der Deposition ermöglicht wird, darf erwartet werden, dass die Daten aus dem Moosmonitoring die Präzision künftiger Depositionsmodellierung erhöhen (Ilyin 2009). Dies ist erforderlich, denn die intrinsische Unsicherheit der Modellierung der Gesamtdeposition mit den EMEP-Modellen beträgt für Blei und Cadmium rund $30 \%$ und für Quecksilber rund $40 \%$. Dies bedeutet, dass die Depositionsmodellierungen für das Bundesgebiet räumlich nicht ausreichend präzise sind. Die häufig zum Qualitätsnachweis für das Moosmonitoring erhobene Forderung, die Stoffkonzentrationen im biologischen Material müsse mit der gemessenen oder modellierten Deposition hoch korrelieren, ist nicht sachgemäß, da es sich um Verfahren handelt, die unterschiedliche Rezeptoren berücksichtigen. Die Akkumulation der untersuchten Schadstoffe in den Bodenmoosen reflektiert die im System „Pflanze - Boden“ den durch Depositionen hervorgerufenen, verbleibenden und wirksamen Teil der Schadstoffbelastungen. Hierdurch besitzen die Untersuchungsergebnisse eine deutlich größere Übertragbarkeit auf die Vegetation (einschließlich Nahrungspflanzen) als die Daten technischer Messungen.

In Frankreich und Deutschland werden die höchsten MMI-Werte für Gegenden mit hohem Flächenanteil urbaner Landnutzung ermittelt. Während in Frankreich auch eine positive Korrelation der MMI (2005) mit der Höhenlage über NN gefunden wurde (Holy et al. 2009b), konnte dies von Schröder et al. (2009) anhand der deutschlandweiten Daten von 2005 ebenso wenig bestätigt werden wie von Pesch et al. (2007b) auf Grundlage der Daten aus dem Jahr
2000. Die deutschen Befunde sind im Einklang mit Coşkun et al. (2005) und Schmidt-Grob et al. (1991). Gerdol et al. (2002) sowie Sucharova und Suchara (2004) berichten über negative Korrelationen zwischen Metallkonzentrationen in Moosen und der Höhenlage der beprobten Orte. Die französischen Befunde werden hingegen von Šoltès (1998), Zechmeister (1994, 1995), Zechmeister et al. (2003) sowie von Gerdol und Bragazza (2006) gestützt.

Wie für das Bundesgebiet, so zeigt sich auch für Niedersachsen, dass eine räumlich hinreichend dichte Beprobung nicht allein anhand von einer Moosart, dem Rotstängelmoos (Pleurozium schreberi) erfolgen kann, sodass andere Moosarten mit ähnlichen Akkumulationseigenschaften (Scleropodium purum, Hypnum cupressiforme) hinzugezogen wurden. Siewers et al. (2000) und Harmens et al. (2008) kommen zu dem Schluss, dass die Metallakkumulation je nach Metall und Region artenspezifisch sein kann. Analysen der deutschen Moosmonitoringdaten der Jahre 1990, 1995, 2000 und 2005 konnten dies teilweise belegen (Kleppin et al. 2008a, Schröder et al. 2008, 2009). Für Frankreich wurde offenbar, dass Pleurozium schreberi und Scleropodium purum geringere Metallgehalte aufweisen als Hypnum cupressiforme. Hierbei ist jedoch zu beachten, dass die miteinander verglichenen Moosarten nicht jeweils von denselben, sondern von unterschiedlichen Sammelorten stammen und die in ihnen gemessenen Stoffgehalte folglich nicht ursächlich mit der Moosart, sondern mit abweichenden Depositionsverhältnissen und anderen akkumulationsrelevanten Merkmalen der unterschiedlichen Sammelorte zusammenhängen können. Leblond und Rausch de Traubenberg (2006, 2007) weisen für jeweils an einem von 15 Standorten gesammelte Proben der Arten Scleropodium purum, Hypnum cupressiforme und Thuidium tamariscinum signifikant unterschiedliche Metallgehalte nach. Doch die Arten mit den höchsten Gehalten waren nicht bei allen Elementen dieselben, die Akkumulationseigenschaften sind also art- und stoffspezifisch. Artenspezifische Unterschiede werden auch von Castello (2007), Olajire (1998) sowie Zechmeister et al. (2003) berichtet. Rühling und Tyler (1968) sowie Ashmore et al. (2000) konnten dies jedoch nicht bestätigen. Eine geringe Dichte der Moosdecke wurde in Frankreich und Deutschland als weiterer wichtiger Standortfaktor für die Metallakkumulation identifiziert (Holy et al. 2009b; Pesch et al. 2007b).

Zur Beseitigung derart systematischer Fehler würde die Umrechnung der Metallkonzentrationen auf jeweils eine Moosart allerdings Unschärfen erzeugen. Zudem fallen sie gegenüber den vorhandenen Unterschieden der regional variierenden Metallkonzentrationen nicht ins Gewicht, weshalb hierauf verzichtet wurde. Weitere räumliche und standörtliche Einflüsse auf die Metallkonzentrationen lassen sich durch die statistische Untersuchung mit Entscheidungsbaumverfahren offenlegen (CART-Analyse, Kap. 3). 


\section{Schlussfolgerungen}

Physikalische Messnetze (z.B. UBA Luftmessnetz, ICP Forest Level II, Umweltprobenbank) können nur an wenigen Orten Daten erheben, liefern gegenüber dem Moosmonitoring jedoch zeitlich besser aufgelöste Informationen. Die hohe räumliche Auflösung der Daten aus dem Moosmonitoring ist nützlich, um auch unerwartete Belastungen zu identifizieren. Es liefert für die räumliche Modellierung derzeit die einzige Datengrundlage für eine Validierung der räumlichen Differenzierung auf kontinentaler, nationaler und regionaler Ebene.

Im Gegensatz zu physikalisch-chemischen Messungen gibt das Moosmonitoring Auskunft darüber, welche Stoffe und in welcher Höhe diese am Wirkort/Schutzgut Pflanze (Moos) ankommen. Zudem ist die Bioakkumulation von Stoffen in der Umwelt für die ökotoxikologische Bewertung der Erheblichkeit von Stoffeinträgen z. B. in FFH-Gebieten und landwirtschaftliche Kulturen aussagekräftiger als die gemessene atmosphärische Deposition. Das Moosmonitoring liefert nicht nur eine Bestimmung der Umweltkonzentration von Stoffen, sondern immissionsschutzrechtlich relevante Beiträge zur Wirkungsermittlung; denn die Schadstoffanreicherung in einem Organismus ist die Vorstufe einer potenziellen toxischen Wirkung. Insofern ist das passive Monitoring mit Moosen insbesondere im Hinblick auf Vorsorgemaßnahmen wichtig. Technische Depositionsmessungen und die Bestimmung der Akkumulation von Stoffen in Ökosystemkompartimenten resultieren in Informationen zu verschiedenen, aufeinander folgenden Schritten in der Prozesskette Emission, Immission sowie Akkumulation/ Wirkung. Das Moosmonitoring ist dabei ein wichtiges Bindeglied zu den biologischen Wirkungen. Ein Schwermetallindikator auf Grundlage der Moosmonitoringdaten liegt für einzelne Naturräume, Bundesländer und Schutzgebiete vor.

\section{Empfehlungen und Ausblick}

Die flächenrepräsentative Bestimmung der luftbürtigen Schwermetallbelastungen und somit eine zuverlässige und räumlich hinreichend aufgelöste Identifikation von Belastungsgebieten ist derzeit nur mit dem Moosmonitoring möglich. Moose sammeln auch bei eingeschränkter Vitalität atmosphärische Inhaltsstoffe. Das gilt selbst dann, wenn sie in weitgehend geschlossenen Räumen als Rezeptoren eingesetzt werden und abgestorben sind (Zechmeister et al. 2006). Untersuchungen zeigen, dass Moos ein sehr gut geeignetes Rezeptormedium für das Indoor- und OutdoorExpositionsmonitoring ist, wodurch sich das Moosmonitoring enger mit dem Humanbiomonitoring verknüpfen lässt. Die räumlich differenzierte Erfassung der Beziehungen zwischen der inneren Exposition von Probanden durch
Humanbiomonitoring einerseits - beispielsweise von Cd in der menschlichen Nahrungskette (EFSA 2009) - sowie der äußeren Exposition innerhalb und außerhalb von Gebäuden durch Umweltmonitoring andererseits fehlt bislang (Pesch et al. 2009). Ziel sollte es sein, eine Biomonitoringmethode für die Langzeiterfassung organischer und anorganischer Schadstoffe mit dem Rezeptor Moos zu entwickeln und zu erproben, die über ein breites Spektrum von Stoffen und Klimabedingungen im Indoor- und Outdoor-Monitoring einsetzbar ist und die Beziehungen zwischen innerer Exposition und äußerer Exposition (Indoor, Outdoor) zuverlässig erfasst (Zechmeister et al. 2007).

\section{Literatur}

Ashmore M, Bell S, Fowler D, Hill M, Jordan C, Nemitz E, Parry S, Pugh B, Reynolds B, Williams J (2000) Survey of the UK metal content of mosses 2000. Part II of EPG 1/3/144 final contract report. Development of a critical load methodology for toxic metals in soils and surface waters: stage II. University of Bradford, Bradford, UK

Bealey W, Cape JN, Leith ID, Long S, Kinnerlsey RP (2008a) Air quality outcomes in pollution regulation: strengths, limitations and potential. Science Report SC030175/SR1, CEH Project Number: C02600. Environment Agency, Bristol, pp 1-47

Bealey WJ, Long S, Spurgeon DJ, Leith I, Cape JN (2008b) Review and implementation study of biomonitoring for assessment of air quality outcomes. Science Report SC030175/SR2. Environment Agency, Bristol, pp 1-170

Breimann L, Friedmann JA, Olshen RA, Stone CJ (1984) Classification and regression Trees. Wadsworth, Belmont, CA

Castello M (2007) A comparison between two moss species used as transplants for airborne trace element biomonitoring in NE Italy. Environ Monit Assess 133:267-276

Coşkun M, Frontasyeva MV, Steinnes E, Cotuk AY, Pavlov SS, Coşkun M, Sazonov AS, Cayir A, Belivermis M (2005) Atmospheric Deposition of Heavy Metals in Thrace Studied by Analysis of Moss (Hypnum cupressiforme). B Environ Contam Tox 74:201-209

Dämmgen U (2005) Bestimmung von Ammoniak-Einträgen aus der Luft und deren Wirkungen auf Waldökosysteme (ANSWER-Projekt). Landbauforschung Völkenrode Sonderheft 279:1-113

EFSA (European Food Safety Authority) (2009) Cadmium in food. Scientific opinion of the Panel on Contaminants in the Food Chain (Question No EFSA-Q-2007-138). The EFSA Journal 980:1-139

Forster E-M, Matthies M, Brüggemann R (1993) Optimierung eines Bioindikator-Meßnetzes mit geostatistischen Methoden und einem geografischen Informationssystem. Umweltwiss Schadst Forsch 5:286-294

Funk W, Dammann V, Donnevert G (2006) Quality assurance in analytical chemistry. Applications in environmental, food and materials analysis, biotechnology and medical engineering, 2nd edn. Wiley-VCH, Weinheim

Genßler L, Rademacher J, Rammert U (2001) Arbeitskreis der Landesanstalten und -ämter. Konzeption der künftigen Aufgabenbereiche. Umweltwiss Schadst Forsch 13:1-4

Gerdol R, Bragazza L (2006) Effects of altitude on element accumulation in alpine moss. Chemosphere 64:810-816

Gerdol R, Bragazza L, Marchesini R (2002) Element concentrations in the forest moss Hylocomium splendens: variation associated with altitude, net primary production and soil chemistry. Environ Pollut 116:129-135 
Harmens H (2005) Monitoring of atmospheric deposition in Europe using bryophytes. Monitoring manual 2005/2006 survey. Bangor, UK

Harmens H, Norris D et al. (2008) Spatial and temporal trends in heavy metal accumulation in mosses in Europe (1990-2005). Programme Coordination Centre for the ICP Vegetation, Centre for Ecology and Hydrology, Environment Centre Wales, Bangor, UK

Herpin U, Siewers U, Markert B, Rosolen V, Breulmann G, Bernoux M (2004) Second German heavy-metal survey by means of mosses, and comparison of the first and second approach in Germany and other European countries. Env Sci Pollut Res 11:57-66

Holy M, Pesch R, Schröder W, Harmens H, Alber R, Aleksiayenak Y, Blum O, Coskun M, Dam M, de Temmermann L, Frolova M, Frontasyeva M, Grodzińska K, Jeran Z, Kubin E, Kvietkus K, Leblond S, Liiv S, Magnússon S, Maňkovská B, Miodrag K, Rühling Å, Santamaria J, Steinnes E, Suchara I, Szymon K, Thöni L, Urumov V, Yurukova L, Zechmeister (2009a) Europe-wide analysis of factors influencing the spatial variation of cadmium, lead and mercury concentrations in mosses. In: Harmens H, Mills G, Menichino N, Bender J, Weigel H (eds) 22nd Task Force Meeting of the ICP Vegetation, 2-5 February 2009, Braunschweig. ICP Vegetation Programme Coordination Centre, Centre for Ecology and Hydrology, Bangor, UK, p 26

Holy M, Leblond S, Pesch R, Schröder W (2009b) Assessing spatial patterns of metal bioaccumulation in France by means of an exposure index. Environ Sci Pollut Res 16(5):499-507

Holy M, Pesch R, Schröder W, Ilyin I, Harmens H, Alber R, Aleksiayenak Y, Blum O, Coskun M, Dam M, de Temmermann L, Frolova M, Frontasyeva M, Grodzińska K, Jeran Z, Kubin E, Kvietkus K, Leblond S, Liiv S, Magnússon S, Maňkovská B, Miodrag K, Rühling Å, Santamaria J, Steinnes E, Suchara I, Szymon K, Thöni L, Urumov V, Yurukova L, Zechmeister (2009c) Comparison of Europe-wide data on metal bioaccumulation in mosses with modelled emission and deposition data. Atmosph Environ (submitted)

Ilyin I (2009) Modelled EMEP heavy metal deposition vs moss measurements: Evaluation of spatial patterns and log-term temporal trends. In: Harmens H, Mills G, Menichino N, Bender J, Weigel H (eds) 22nd Task Force Meeting of the ICP Vegetation, 2-5 February 2009, Braunschweig. ICP Vegetation Programme Coordination Centre, Centre for Ecology and Hydrology, Bangor, UK p 27

Johnston K, Ver Hoef JM, Krivoruchko K, Lucas N (2001) Using ArcGIS Geostatistical Analyst. ESRI, Redlands

Keil M, Kiefl R, Strunz G (2005) CORINE Land Cover 2000 Germany. Final Report. German Aerospace Center, German Remote Sensing Data Center, Oberpfaffenhofen

Kleppin L, Pesch R, Schröder W (2008a) CHAID-Models on boundary conditions of metal accumulation in mosses collected in Germany 1990, 1995 and 2000. Atmos Environ 42:5220-5231

Kleppin L, Schröder W, Pesch R, Schmidt G (2008b) Entwicklung und Erprobung einer Metadaten- und WebGIS-Applikation für das Expositionsmonitoring mit Moosen in Deutschland. Ein Beitrag zum LTER-Netzwerk. Umweltwiss Schadst Forsch 20:38-48

Klimmek S (2003) Charakterisierung der Biosorption von Schwermetallen an Algen. Dissertation TU Berlin, Berlin, S 1-142

Kostka-Rick R, Leffler US, Markert B, Herpin U, Lusche M, Lehrke J (2001) Biomonitoring zur wirkungsbezogenen Ermittlung der Schadstoffbelastung in terrestrischen Ökosystemen. Konzeption, Durchführung und Beurteilungsmaßstäbe im Rahmen von Genehmigungsverfahren. Umweltwiss Schadst Forsch 12:5-12

Leblond S, Rausch de Traubenberg C (2006) Etude des retombées atmosphériques de métaux en France. Estimation par dosage dans des mousses. Campagne 2006 du dispositif BRAMM (Biosurveillance des Retombées Atmosphériques Métalliques par les Mousses), Marché ADEME N ${ }^{\circ}$ 0562C0036, 1er Rapport Intermédiaire, Paris

Leblond S, Rausch de Traubenberg C (2007) Etude des retombées atmosphériques de métaux en France. Estimation par dosage dans des mousses. Campagne 2006 du dispositif BRAMM (Biosurveillance des Retombées Atmosphériques Métalliques par les Mousses) - Paris. (Marché ADEME N 0562C0036, 2ième Rapport Intermédiaire)

Matheron G (1965) Les variables régionalisées et leur estimation. Masson, Paris

Mohr K (1999) Passives Monitoring von Stickstoffeinträgen in Kiefernforsten mit dem Rotstengelmoos (Pleurozium schreberi (Brid) Mitt). Umweltwiss Schadst Forsch 11:267-274

Mohr K (2007) Biomonitoring von Stickstoffimmissionen. Möglichkeiten und Grenzen von Bioindikationsverfahren. Umweltwiss Schadst Forsch 19(4):255-264

Olajire A (1998) A survey of heavy metal deposition in Nigeria using the moss monitoring method. Environ Int 24(8):951-958

Pesch R (2003) Geostatistische und multivariat-statistische Analyse des Moos-Monitorings 1990, 1995 und 2000 zur Ableitung von Indikatoren für die Bioakkumulation atmosphärischer Metalleinträge in Deutschland. Dissertation, Hochschule Vechta

Pesch R, Schröder W (2006) Integrative exposure assessment through classification and regression trees on bioaccumulation of metals, related sampling site characteristics and ecoregions. Ecol Inf $1: 55-65$

Pesch R, Schmidt G, Schröder W, Aden C, Kleppin L, Holy M (2007a) Development, implementation and application of the WebGIS MossMet. In: Tochtermann K, Scharl A (eds) The geospatial web. How geo-browsers, social software and the Web 2.0 are shaping the network society. Springer, London, pp 191-200

Pesch R, Schröder W, Genßler L, Göritz A, Holy M, Kleppin L, Matter Y (2007b) Moos-Monitoring 2005/2006: Schwermetalle IV und Gesamtstickstoff. Umweltforschungsplan des Bundesministers für Umwelt, Naturschutz und Reaktorsicherheit. FuE-Vorhaben 20564 200, Abschlussbericht, im Auftrag des Umweltbundesamtes. Berlin

Pesch R, Schröder W, Dieffenbach-Fries H, Genßler L, Kleppin L (2008) Optimierung des Moos-Monitoring-Messnetzes in Deutschland. Umweltwiss Schadst Forsch 20:49-61

Pesch R, Schmidt G, Schröder W, Conrad A, Kolossa-Gehring M, Feigenspan S, Utermann J (2009) Das Potenzial raumbezogener Daten im Human-Biomonitoring am Beispiel des Kinder-Umwelt-Surveys. In: Strobl J, Blaschke T, Griesebner G (Hrsg) Angewandte Geoinformatik 2009. Wichmann, Heidelberg, pp 248-257

Poikolainen J (2004) Mosses, epiphytic lichens and tree bark as biomonitors for air pollutants - specifically for heavy metals in regional surveys. Dissertation, University of Oulu

Rühling A, Tyler G (1968) An ecological approach to the lead problem. Botaniska Notiser 121:321-343

Rühling A, Tyler G (1969) Ecology of heavy metals - a regional and historical study. Botaniska Notiser 121:248-259

Rühling A, Tyler G (1970) Sorption and retention of heavy metals in the woodland moss Hylocomium splendens (Hedw.) Br. Et Sch Oikos 21:248-342

Schmidt-Grob I, Thöni L, Hertz J (1991) Übersicht über die Deposition von Arsen, Blei, Cadmium, Chrom, Cobalt, Eisen, Kupfer, Molybdän, Nickel, Quecksilber, Schwefel, Thallium, Vanadium und Zink in der Schweiz mit Hilfe von Moosen als Biomonitoren. Forschungsstelle für Umweltbeobachtung, Birmensdorf, Schweiz

Schröder W, Schmidt G (2000) Raumgliederung für die Ökologische Umweltbeobachtung des Bundes und der Länder. Umweltwiss Schadst Forsch 12:237-243

Schröder W, Pesch R (2004) The 1990, 1995 and 2000 moss monitoring data in Germany and other European countries Trends and statistical aggregation of metal accumulation indicators. Gate Environ Health Sci: $1-25$

Schröder W, Pesch R (2005) Time series of metals in mosses and their correlation with selected sampling site-specific and ecoregional characteristics in Germany. Env Sci Pollut Res 12:159-167 
Schröder W, Garbe-Schönberg CD, Fränzle O (1991) Die Validität von Umweltdaten. Kriterien für ihre Zuverlässigkeit: Repräsentativität, Qualitätssicherung und -kontrolle. Umweltwiss Schadst Forsch 3:237-241

Schröder W, Pesch R, Schmidt G (2004) Soil monitoring in Germany Spatial representativity and methodical comparability. J Soils Sediments 4:49-58

Schröder W, Englert C, Pesch R, Zechmeister HG, Thöni L, Suchara I, Maňkovská B, Jeran Z, Harmens H, Grodzinska K, Alber R (2008a) Metallakkumulation in Moosen: Standörtliche und regionale Randbedingungen des Biomonitoring von Luftverunreinigungen. Umweltwiss Schadst Forsch 20:120-132

Schröder W, Hornsmann I, Pesch R, Schmidt G, Fränzle S, Wünschmann S, Heidenreich H, Markert B (2008b) Moos-Monitoring als Spiegel der Landnutzung? Stickstoff- und Metallakkumulation in Moosen zweier Regionen Mitteleuropas. Umweltwiss Schadst Forsch 20:62-74

Schröder W, Pesch R, Matter Y, Dieffenbach-Fries H, Genßler L (2009) Trend der Schwermetall-Bioakkumulation 1990 bis 2005 in Deutschland. Umweltwiss Schadst Forsch 21 (im Druck)

Siewers U, Herpin U, Straßburger S (2000) Schwermetalleinträge in Deutschland. Moos-Monitoring 1995, Teil 2. Geolog Jahrb, Sonderhefte SD 3. Bornträger, Stuttgart

Šoltès R (1998) Correlation between altitude and heavy metal deposition in the Tatra Mountains (Slovakia). Biologia 53:85-90

Steinnes E, Rühling Å, Lippo H, Mäkinen A (1997) Reference materials for large-scale metal deposition surveys. Accredit Qual Assur 2(5):243-249

Sucharova J, Suchara I (2004) Bio-monitoring the atmospheric deposition of elements and their compounds using moss analysis in the Czech Republic. Results of the international bio-monitoring pro- gramme UNECE ICP-Vegetation 2000. Part I: Elements required for the bio-monitoring programme. Acta Pruhoniciana 77

Tyler G. (1990) Bryophytes and heavy metals: a literature review. Bot J Linnean Soc 104:231-253

Wappelhorst O, Korhammer S, Leffler US, Markert B (2000) Ein Moosbiomonitoring zur Ermittlung atmosphärischer Elementeinträge in die Euroregion Neiße (D, PL, CZ) Umweltwiss Schadst Forsch 12(4): 191-200

Webster R, Oliver MA (2001) Geostatistics for environmental scientists. Wiley, Chichester New York Weinheim Brisbane Singapore Toronto

Zechmeister HG (1994) Biomonitoring der Schwermetalldepositionen mittels Moosen in Österreich. Monogr Umweltbundesamt 42:1-168

Zechmeister HG (1995) Correlation between altitude and heavy metal deposition in the Alps. Environ Pollut 89:73-80

Zechmeister HG, Grodzinska K, Szarek-Lukaszewska G (2003) Bryophytes. In: Markert BA, Breure AM, Zechmeister HG (eds) Bioindicators \& biomonitors. Principles, concepts and applications. Elsevier, Amsterdam, pp 329-375

Zechmeister HG, Dullinger S, Hohenwallner D, Riss A, Hanus-Illnar A, Scharf S (2006) Pilot study on road traffic emissions (PAHs, heavy metals) measured by using mosses in a tunnel experiment in Vienna, Austria. Environ Sci Pollut Res 13:398-405

Zechmeister HG, Köllensperger G, Hann S, Hanus-Illnar A, Scharf S, Schneider J, Neuberger M, Moshammer H, Hohenwallner D, Schierl R, Schröder W, Schmidt G, Pirintsos SA, Loppi S, Gaggi C, Nante N, Namiesnik J, Zygmunt B, Jeran Z, Perharic L, Künzli N, Frontasyeva M, Lyapunov S, Ilchenko I, Harmens H (2007) INDOMO. New tool for monitoring indoor pollutants with health relevance across Europe. Wien (unpublished) 